\title{
PLASMA POWER EFFECT ON THE SURFACES OF A QUARTZ CRYSTAL DURING ETCHING USING TETRAFLUOROETHANE GAS
}

\author{
Masruroh $^{1 *}$, Mahardika Auditia Hanif ${ }^{1}$, Setyawan P. Sakti ${ }^{1}$, Dionysius J. D. H. Santjojo ${ }^{1}$ \\ ${ }^{1}$ Department of Physics, Faculty of Mathematics and Natural Sciences, Brawijaya University, \\ Jl. Veteran, Malang 65145, Indonesia
}

(Received: January 2017 / Revised: May 2017 / Accepted: November 2017)

\begin{abstract}
The performance of a quartz crystal microbalance (QCM) biosensor can be enhanced by patterning the surface of the $\mathrm{SiO}_{2}$ substrate. In this study, the patterning was realized by a plasma etching process. The etching of the $\mathrm{SiO}_{2}$ was carried out using a tetrafluoroethane $\left(\mathrm{CH}_{2} \mathrm{FCF}_{3}\right)$ plasma. The plasma was generated by applying power from a generator. The generator used in this research was a low frequency $40 \mathrm{kHz}$ plasma generator. The generator was equipped with automatic matching circuits, which ensured the stability of plasma power during the experiments. The specimens were produced with a power ranging from 40 watts to 120 watts for 1 hour. The pressure of the chamber was fixed at 1 Torr. The processing gas for this study was a commercial $\mathrm{CH}_{2} \mathrm{FCF}_{3}$ gas. The flow rate of the gas was $20 \mathrm{ml} / \mathrm{min}$. The purpose of this research was to study the effect of plasma power on the etching rate and the anisotropy of the etched $\mathrm{SiO}_{2}$ surface. The etching rate and the anisotropy strongly correlate with the quality of patterning. Measurement and observation of the etched $\mathrm{SiO}_{2}$ surface were carried out using an optical microscope and a TMS-1200 (Topography Measurement System). The optical microscope was used to determine the etched area from the unetched one, while the TMS was utilized to obtain the thickness and the surface profile. The results show the highest etching rate, i.e., $17.90 \mathrm{~nm} / \mathrm{min}$, was obtained by applying a plasma power of $100 \mathrm{watts}$. The rate demonstrated a relatively slow etching process due to a complex mixture of fluorine $(\mathrm{F})$ and the $\mathrm{CH}_{2} \mathrm{FCF}_{2}$ compound. This slow etching rate is preferable for controlling nano-profiles of the pattern. Furthermore, the applied power also had an effect on the anisotropy of the etched profile, and the results of this research show that the best anisotropic coefficient, i.e., $4.8 \times 10^{-2}$, occurred in the process with an optimized 110-watt power. The anisotropy was defined as the ratio of the vertical etching rate and the horizontal etching rate. This ratio is important in determining the quality of the profile of the patterned QCM.
\end{abstract}

Keywords: Anisotropic coefficient; Etching rate; Low-frequency generator; Tetrafluoroethane gas

\section{INTRODUCTION}

A quartz crystal microbalance (QCM) sensor is a device which uses the piezoelectric effect to measure a very small mass change by calculating the change in frequency of a quartz crystal resonator. The resonant frequency of the crystal is determined by crystal parameters. In the biosensor applications, the QCM sensitivity depends on the thickness of the crystal as well as the acoustic impedance of the additional layer on top of it (Masruroh et al., 2016). In general, a

\footnotetext{
*Corresponding author's email: ruroh@ub.ac.id, Tel: +62-341-554403, Fax: +62- 341-554403

Permalink/DOI: https://doi.org/10.14716/ijtech.v8i8.721
} 
thinner crystal produces higher sensitivity. However, a thinner crystal also leads to instability of the crystal oscillation. Researchers have, therefore, been working to improve the stability of the sensor. One of the methods proposed involves profiling or patterning the surface of the crystals (Goyal, 2006). Profiles or patterns on the surface can be optimized to suppress the out-of-plane flexural vibrations in the sensor.

The surface patterning can be created by using an etching method. Some researchers have reported that a plasma etching process was used to make a patterned layer on a quartz crystal resonator to improve its sensitivity for biochemical sensor applications (Lieberman \& Lichtenberg, 2005; Goyal, 2006; Fridman, 2008). Plasma etching is a process used to remove material by ion bombardment in a reactive plasma onto an exposed surface. Plasma etching of $\mathrm{SiO}_{2}$ can usually be realized by using a certain type of gas, such as $\mathrm{SiF}_{4}$ or other gases containing fluorine (Li et al., 2002; Lieberman \& Lichtenberg, 2005). There are many fluorinecontaining gases available in the market. However, many of these are not environmentally friendly. Considering the environmental impact of the process, this study utilizes tetrafluoroethane $\left(\mathrm{CH}_{2} \mathrm{FCF}_{3}\right)$ gas as the source of plasma. The effectiveness of the etching process using $\mathrm{CH}_{2} \mathrm{FCF}_{3}$ has not been established, as the conventional usage of the gas is in air conditioning and refrigeration technology. In this study, the influence of the plasma power on the etching rate and the anisotropy of the etched $\mathrm{SiO}_{2}$ surface was investigated.

\section{METHODS}

The $10 \mathrm{MHz}$ AT-cut quartz crystal with silver (Ag) electrode used in this experiment was purchased from Greatmicro Electronics. Before the etching process, the $\mathrm{SiO}_{2}$ crystal was cleaned using distilled water in the ultrasonic cleaner bath; next the Ag electrode was removed using $\mathrm{HNO}_{3}$ solution. Then the entire surface of the sample, except for the middle surface, was covered using Kapton tape masking, in order to protect the unetched area. Subsequently, the sample was placed in the plasma chamber. A commercial $\mathrm{CH}_{2} \mathrm{FCF}_{3}$ gas was used to process gas in the plasma etching process. The plasma etching system used in this study was operated with a low frequency $40 \mathrm{kHz}$ plasma generator. The generator was equipped with an automatic matching circuit which maintained the stability of the plasma during the experiments. The reaction chamber was evacuated by means of a rotary pump. The flow rate of the gas used in this study was set to $20 \mathrm{ml} / \mathrm{min}$. The etching process was carried out at the chamber pressure of 1 Torr, and samples were treated by plasma with a power ranging from 40 watts to 120 watts for 1 hour for each variation of power exerted.

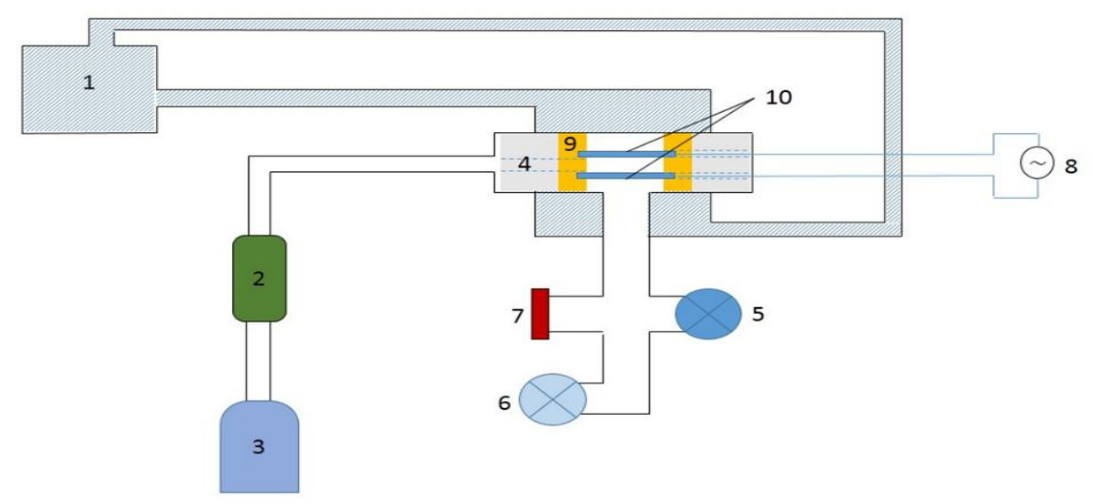

Figure 1 Design of plasma etching system: (1) Water reservoir; (2) Gas flow meter; (3) Gas container $\left(\mathrm{CH}_{2} \mathrm{FCF}_{3}\right)$; (4) Insulator; (5) Exhaust valve; (6) Venting valve; (7) Pressure gauge; (8) RF Generator; (9) Ceramic spacer; (10) Electrodes 
After being treated by plasma etching, the samples were characterized using a white light profilometer (surface profilometer polytec TMS $1200 \mu$ Surf) to determine the depth of etched surface.

The etching rate was calculated using the following equation:

$$
E=\frac{z}{t}
$$

where $z$ is the depth (nm) and $t$ is the etching duration time.

The anisotropy coefficient is determined by observing the cross-section of the etched sample shown in Figure 2 and calculated using the following equations:

$$
a_{h}=\frac{E_{v}}{E_{h}}
$$

where $a_{h}$ is the anisotropic coefficient, $E_{v}$ is the vertical etching rate $(\mathrm{nm} / \mathrm{min})$, and $E_{h}$ is the horizontal etching rate $(\mathrm{nm} / \mathrm{min})$.

$$
a_{h}=\frac{d}{\delta}
$$

where $d$ represents the vertical etching rate $E_{v}$ and $\delta$ represents the horizontal etching rate $E_{h}$ (Lieberman \& Lichtenberg, 2005).

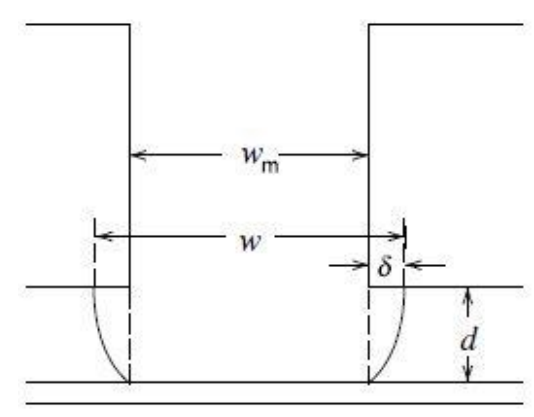

Figure 2 Cross-section of an etched sample

\section{RESULTS AND DISCUSSION}

The etching rate was calculated by determining the depth $(\mathrm{z})$ of the etched surface using TMS. The TMS results in Figure 3 show color bars which indicate the height of the two different regions, i.e., the unetched and etched regions, respectively. The blue colors indicate areas with a shallow surface (the unetched area), while the red colors indicate the deeper surfaces (the etched area). The depth (z) can be obtained by subtracting the measured heights at points 1 and 2 on the line profile graph. The etching rate (E) was calculated using Equation 1. The value of $z$ and $\mathrm{E}$ for various amounts of power are summarized in Table 1 and visualized in Figure 4. 

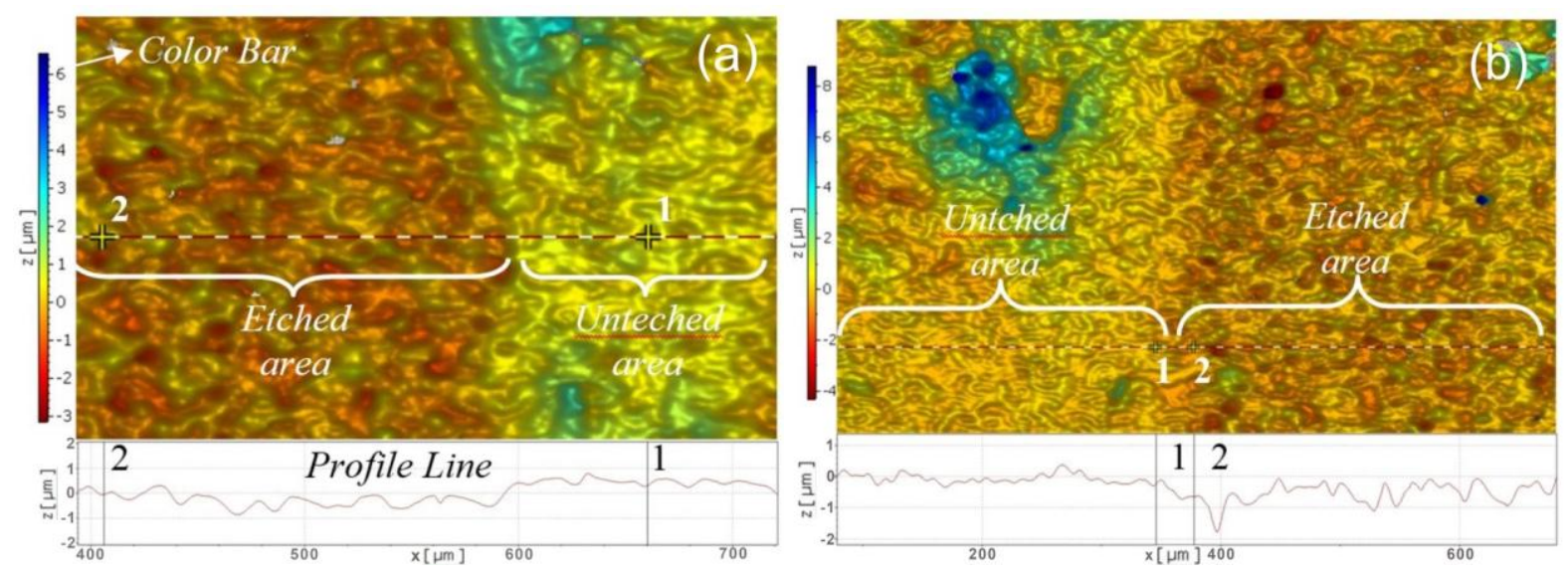

Figure 3 2-D TMS micrographs of $\mathrm{SiO}_{2}$ after the etching process: 1 indicates the etched area, 2 indicates the unetched area; (a) 40 watts and (b) 60 watts

Table 1 Effect of power on depth, etching rate, and anisotropy coefficient of the etching process using a quartz crystal and tetrafluoroethane

\begin{tabular}{ccc}
\hline Power (watts) & Depth of etched surface, $z(\mathrm{~nm})$ & Etching rate, $E(\mathrm{~nm} / \mathrm{min})$ \\
\hline 40 & 715.14 & 11.98 \\
60 & 780.48 & 13.00 \\
80 & 976.02 & 16.26 \\
100 & 1074.36 & 17.90 \\
110 & 1017.26 & 16.96 \\
\hline
\end{tabular}

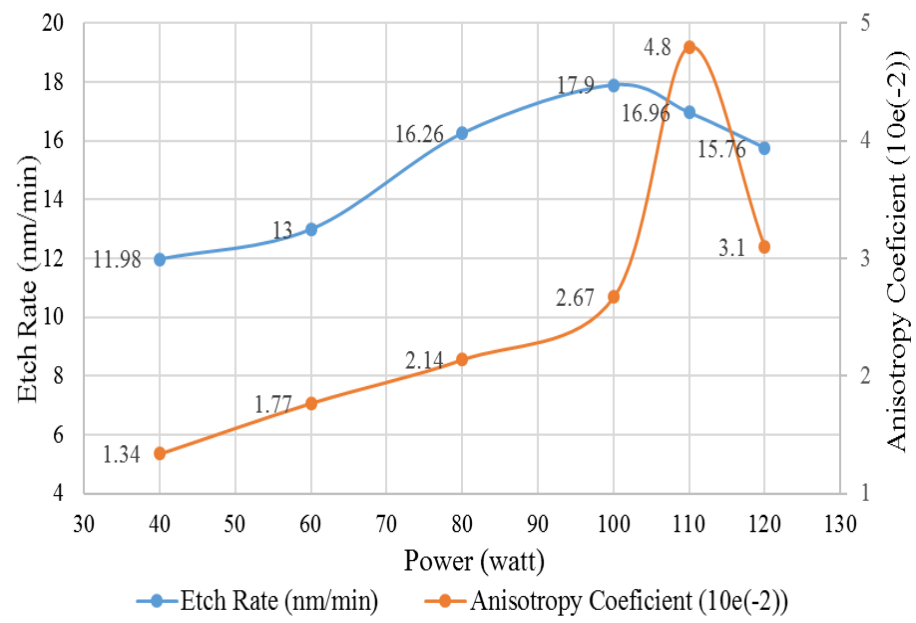

Figure 4 The effect of power on the etching rate and anisotropy coefficient on the quartz crystal's surface of the etching process using tetrafluoroethane gas

From Table 1 and Figure 4, it can be seen that the etching rate and the anisotropy coefficient were clearly affected by the plasma power. The above graph shows that, when the power was increased to 100 watts, this resulted in an increase in the etching rate. In addition to the etching rate, the power also affected the coefficient of anisotropy, which was calculated using Equation 2. The anisotropy coefficient slightly increased up to 100 watts, and drastically increased up to 110 watts; then it decreased at a power of 120 watts. Figures 5a, b and c show the anisotropy profile from the TMS measurements. Inset figures are picked up from the border region of the 
etched and unetched area. The x-axis of each figure represents the horizontal scan of the surface, while the $\mathrm{z}$-axis shows the depth of the profiles.

The power was found to be significantly related to the etching mechanism. Two mechanisms are usually considered in the plasma etching process. The first mechanism is related to particular energy-driven ion etching. The more energy is applied to the system, the more effective the ion bombardment becomes and the more the etching rate increases. The second mechanism is concerned with reactive plasma etching, where the plasma chemically reacts with the atoms on the surface of the processed materials. It can be seen in Figure 4 that the etching rate and also the anisotropy coefficient decreased dramatically at higher power. The etching rate started to decrease at 100 watts, while the anisotropy coefficient began to decrease at 110 watts. Processes in the plasma include complex reactions which produce reactive species. The reactive species in the plasma were deduced by the reaction between gas and electrons, as follows (Lieberman \&Lichtenberg, 2005):

$$
\mathrm{CH}_{2} \mathrm{FCF}_{3}+\mathrm{e}^{-} \rightarrow \mathrm{CH}_{2} \mathrm{FCF}_{2}^{+}+\mathrm{F}+2 \mathrm{e}^{-}
$$

The anisotropic plasma etching process is dominated by the etching process in physics; the ion bombardment process that occurs vertically strikes the material target. Most of the ions that are found within the plasma are positive ions. These positive ions are generated by the presence of dissociative ionization processes that occur between the electron and gas. In this study, the process of dissociative ionization occurred in $\mathrm{CH}_{2} \mathrm{FCF}_{3}$ according to the reaction in Equation 4 . $\mathrm{CH}_{2} \mathrm{FCF}_{2}{ }^{+}$ions are formed as a result of the reaction accelerated by the electric field which is located between two electrodes in the plasma that will strike the target material. At the time of the collision, energy transfer occurs between ions and atoms that are on the surface of the target material. If the surface atoms have greater energy than the binding energy of the material surface, erosion occurs. Therefore, the ion energy is transferred to the surface atoms determined by the etching mechanism.

The pressure of the chamber during the etching in this study was relatively high (1 Torr). At this pressure, the etching process is dominated by a physical collision between the $\mathrm{CH}_{2} \mathrm{FCF}_{2}{ }^{+}$ ions and $\mathrm{F}$ atoms and the $\mathrm{SiO}_{2}$ on the surface of the sample. A decrease in the etching rate and the anisotropy coefficient should be related to a reduction in the collisions or the energy of the ions or atoms during the process. Visual observation of the plasma emission intensity provides some explanation of the mechanisms. Figure 6 shows the conditions of the plasma at a power of 110 watts and 120 watts. At 120 watts of power, the surface of the sample was covered with a bright glow (represented by a dashed rectangle in Figure 6). The bright glow implies the existence of complex reactions involving electrons.

The bright glow in this region usually originates from the transition in the quasi-plasma state. The electrons significantly reduce the plasma density by the following reaction:

$$
\mathrm{CH}_{2} \mathrm{FCF}_{2}^{+}+\mathrm{F}+2 \mathrm{e}^{-} \rightarrow \mathrm{CH}_{2} \mathrm{FCF}_{3}+\mathrm{e}^{-}
$$

This reduction of plasma density, in turn, results in the reduction of ion bombardment. Furthermore, the kinetic energy of the F atoms is also reduced by the collision of this atom with the quasi-plasma. This mechanism is known as screening behavior. This screening behavior was not observed at lower power. 

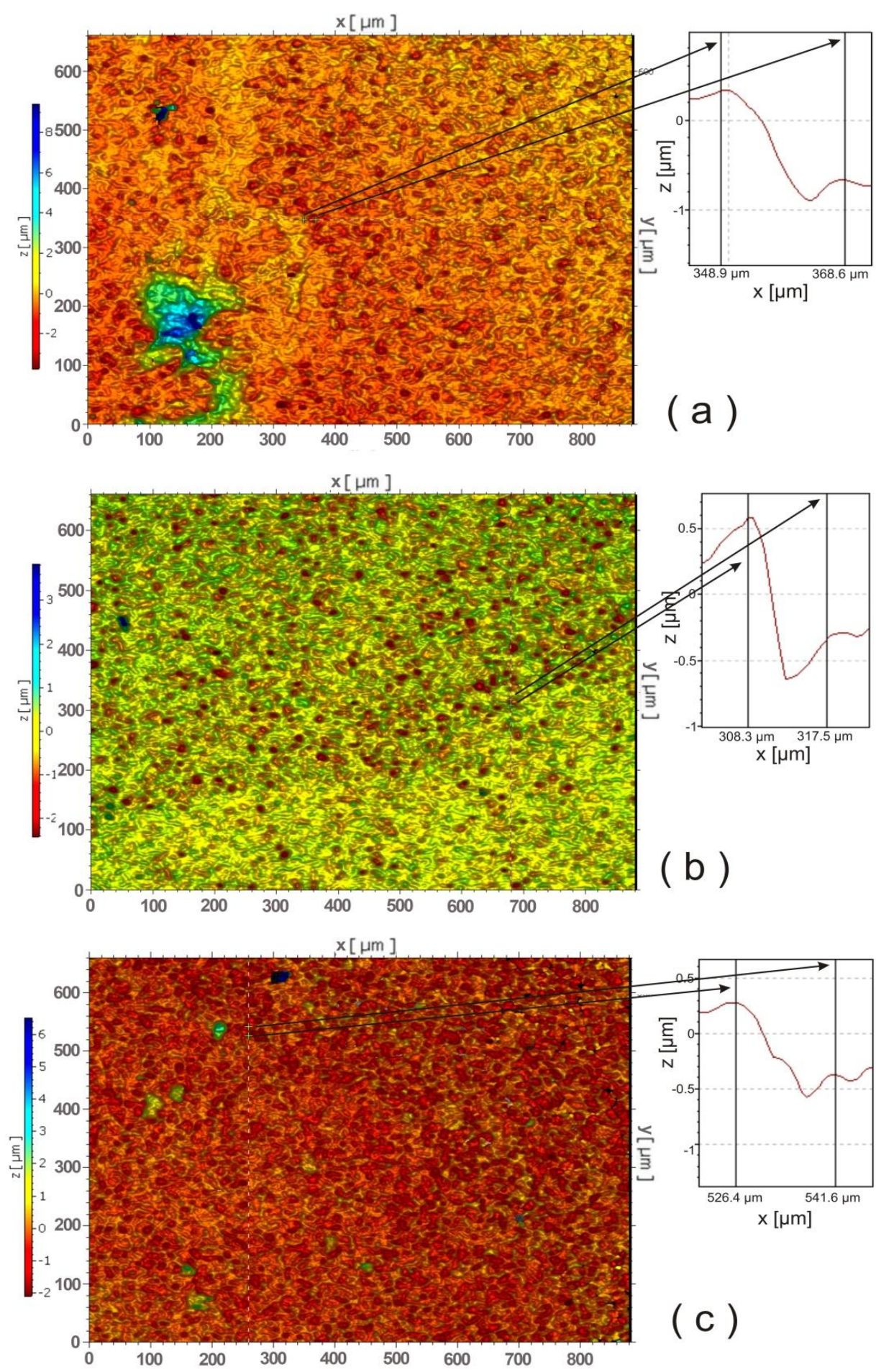

Figure 5 Fine profile of etched samples at various powers: (a) 100 watts; (b) 110 watts; and (c) 120 watts 

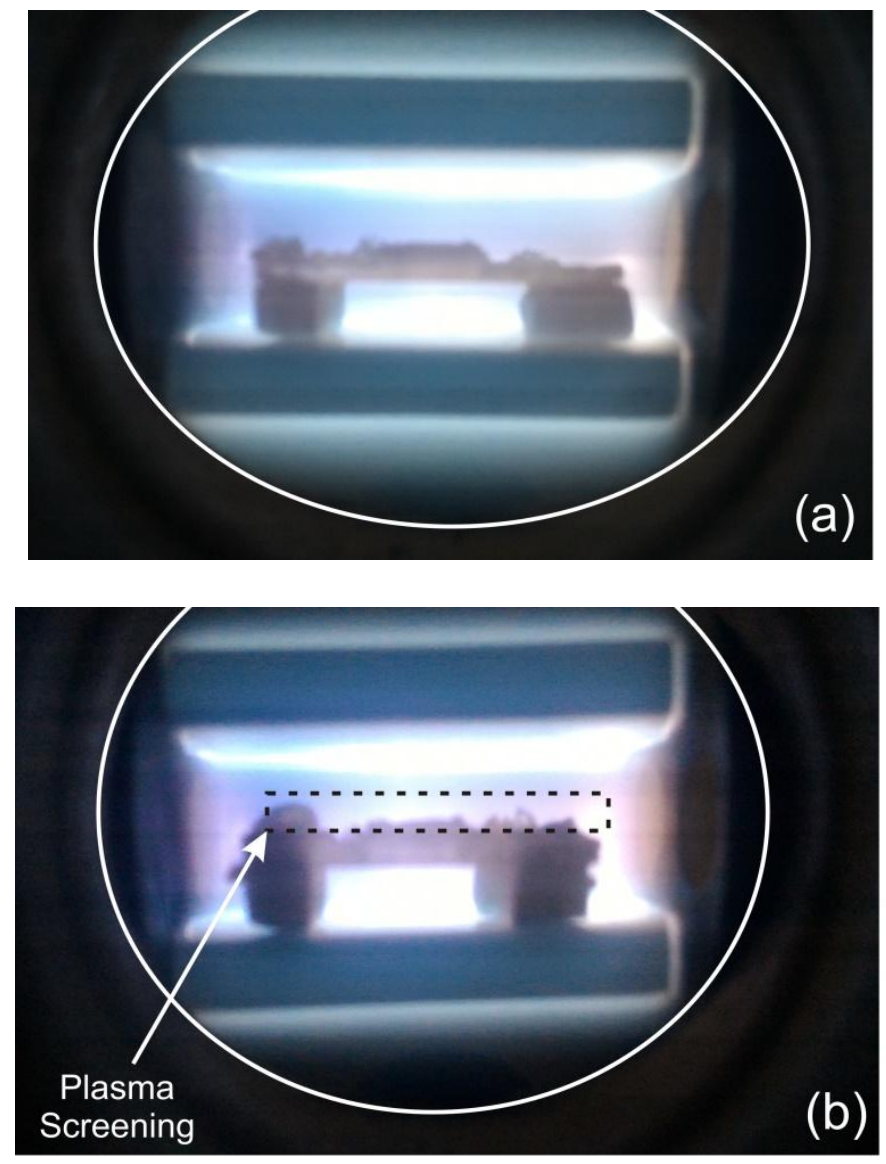

Figure 6 Plasma conditions during the etching process at: (a) 110 watts; and (b) 120 watts

\section{CONCLUSION}

The results of this study demonstrate effective etching of $\mathrm{SiO}_{2}$ by means of a $\mathrm{CH}_{2} \mathrm{FCF}_{3}$ plasma. The depth of etching, etching rate and anisotropy coefficient were observed as a function of power. The optimum power for this process can be found in the range of 100 to 110 watts, where the maximum etching rate and maximum anisotropy coefficient were observed. Power higher than 110 watts resulted in a screening effect which reduced both the etching rate and the anisotropy coefficient.

\section{ACKNOWLEDGEMENT}

This work was financially supported by The Ministry of Research, Technology and Higher Education (RISTEKDIKTI) Republic of Indonesia through LPPM Brawijaya University.

\section{REFERENCES}

Fridman, A., 2008. Plasma Chemistry. Cambridge University Press

Goyal, A., 2006. Ultrasensitive Quartz Crystal Microbalance Integrated with Carbon Nanotube. Thesis Doctor of Philosophy. Graduate School Department of Electrical Engineering, Pennyslvania State University

Li, X., Hua, X., Ling, L., Oehrlein, G.S., Barela, M., Anderson, H.M., 2002. Fluorocarbonbased Plasma Etching of $\mathrm{SiO}_{2}$ : Comparison of $\mathrm{C}_{4} \mathrm{~F}_{5} / \mathrm{Ar}$ and $\mathrm{C}_{4} \mathrm{~F}_{8} / \mathrm{Ar}$ discharges. J. Vac. Sci. Technol. A, Volume 20(6) pp. 2052-2061 
Lieberman, M.A., Lichtenberg, A.J., 2005. Principles of Plasma Discharges and Material Processing. Wiley-Interscience, John Wiley \& Sons, Inc., Publication Hoboken, New Jersey, USA

Masruroh, Djoko, D.J.D.H., Rahayu, S., Sakti, S.P., 2016. Viscoelastic and Morphological Behavior of Stearic Acid Layer on Top of Polystyrene as Immobilisation Matrix for QCM Sensor. Materials Science Forums, Volume 848 pp. 757-762 